\title{
GCU
}

Glasgow Caledonian

University

University for the Common Good

\section{Late diagnosis of chronic liver disease in a community cohort (UK Biobank): determinants and impact on subsequent survival}

Innes, H.; Morling, J.R.; Aspinall, E.A.; Goldberg, D.J.; Hutchinson, S.J.; Guha, I.N.

Published in:

Public Health

DOI:

10.1016/j.puhe.2020.07.017

Publication date:

2020

Document Version

Author accepted manuscript

Link to publication in ResearchOnline

Citation for published version (Harvard):

Innes, H, Morling, JR, Aspinall, EA, Goldberg, DJ, Hutchinson, SJ \& Guha, IN 2020, 'Late diagnosis of chronic liver disease in a community cohort (UK Biobank): determinants and impact on subsequent survival', Public Health, vol. 187, pp. 165-171. https://doi.org/10.1016/j.puhe.2020.07.017

\section{General rights}

Copyright and moral rights for the publications made accessible in the public portal are retained by the authors and/or other copyright owners and it is a condition of accessing publications that users recognise and abide by the legal requirements associated with these rights.

Take down policy

If you believe that this document breaches copyright please view our takedown policy at https://edshare.gcu.ac.uk/id/eprint/5179 for details of how to contact us. 
TITLE: Late diagnosis of chronic liver disease in a community cohort (UK Biobank): determinants and impact on subsequent survival.

\section{AUTHORS:}

Dr Innes $\mathrm{H}^{1,2,3 \#}$; Dr Morling JR ${ }^{3,4 \#}$; Dr Aspinall EA ${ }^{1,2}$; Professor Goldberg DJ ${ }^{2,1}$; Professor Hutchinson $\mathrm{SJ}^{1,2} *$; Dr Guha $\mathrm{IN}^{4} *$

\# joint first authors

* joint senior authors

\section{AFFILIATIONS:}

1. Glasgow Caledonian University; School of Health and Life Sciences; Glasgow, UK.

2. Health Protection Scotland, Glasgow, UK.

3. Division of Epidemiology and Public Health, University of Nottingham, Nottingham, UK

4. NIHR Nottingham Biomedical Research Centre, Nottingham University Hospitals NHS Trust and the University of Nottingham, Nottingham UK.

\section{CORRESPONDING AUTHOR CONTACT DETAILS:}

NAME: Dr Hamish Innes

ADDRESS: Glasgow Caledonian University; Cowcaddens Rd, Glasgow, G4 0BA.

EMAIL: Hamish.innes@gcu.ac.uk

TELEPHONE: 0141-3313915

KEY WORDS: Cirrhosis; late diagnosis; delayed diagnosis; survival; alcohol-related liver disease; NAFLD; prognosis; chronic liver disease

WORD COUNT: 3100 (including introduction; methods; results \& discussion). 
ABSTRACT:

BACKGROUND: Chronic liver disease (CLD) is frequently diagnosed at a late stage when prognosis is poor. We aimed to determine the patient factors associated with a late CLD diagnosis and its subsequent impact on survival in order to support early diagnosis initiatives.

METHODS: We identified participants of UK biobank (UKB) study who developed first-time advanced CLD within 5-years. We identified factors associated with late diagnosis via logistic regression, and used survival analysis to measure the association between late CLD diagnosis and mortality risk.

RESULTS: 725 UKB participants developed first-time advanced CLD event within 5-years. 83\% of cases were diagnosed late. Late diagnosis was associated with aetiology; the odds of late diagnosis were twelve times higher for an individual with alcohol-related liver disease (ArLD) versus viral hepatitis (aOR:12.01;p<0.001).

Cumulative mortality 5-years after incident advanced CLD was 43.4\% (95\%CI:39.6-47.0). Late diagnosis was associated with a higher risk of post- advanced CLD mortality for patients with NAFLD (aHR:2.18; 95\% CI:0.86-5.51; $\mathrm{p}=0.10$ ), but not for other aetiologies.

CONCLUSIONS: Late CLD diagnosis varies according to aetiology, and is highest for patients with ArLD and non-alcoholic fatty liver disease. The association between late diagnosis and postadvanced CLD mortality may also vary by aetiology. 


\section{INTRODUCTION:}

Chronic liver disease (CLD) is a major global public health problem that causes circa two million deaths every year from liver cirrhosis and hepatocellular carcinoma (HCC) ${ }^{1}$. Much of this is increasingly being driven by lifestyle related risk factors such as alcohol consumption and obesity². The issue even more impactful given the poor prognosis from CLD, particularly affecting the working age population ${ }^{3,4}$ therefore giving rise to significant socioeconomic impacts. The true financial costs of CLD are unknown but estimates suggests the indirect costs could be four time that of the direct $\operatorname{costs}^{5}$.Therefore prevention and good management of CLD offers widespread benefits.

A key hallmark of CLD is that it is frequently not diagnosed until after severe irreversible liver morbidity (advanced CLD) -i.e. liver cirrhosis and/or HCC - emerges. For example, data from the Lancet liver disease commission ${ }^{3}$ reported that of more than 4000 individuals admitted to UK hospitals for liver cirrhosis or liver failure between 1996-2012, most (73\%) had not been referred to a specialist liver clinic prior to that event. Furthermore, a UK population based study found that approximately $50 \%$ of incident CLD presentations were with decompensated liver disease ${ }^{4}$, when the prognosis is extremely poor ${ }^{6}$.

Public health policy in the UK is increasingly focused on prevention and early diagnosis ${ }^{7,8}$, but as yet no formal national guidance has been issued on CLD identification. Late CLD diagnosis is associated with a poorer prognosis as patients are unable to access interventions that could have prevented them developing advanced liver disease. Equally late diagnosis may further disadvantage patients as for example patients are less prepared/primed to make appropriate life style changes after advanced liver fibrosis onset versus early diagnosis patients ${ }^{9}$.

Little is presently known about the patient factors associated with the timing of CLD diagnosis; only a handful of studies have explored this issue, and these have been limited to viral hepatitis ${ }^{10,11}$. Previous studies have only examined late diagnosis for patients with viral hepatitis related CLD, Thus far, the factors associated with late diagnosis in a community/primary care population - where most CLD

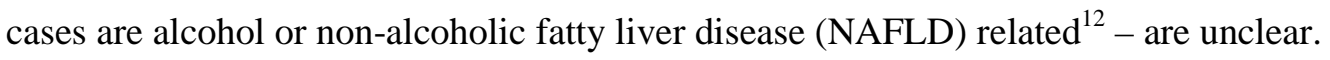

The aim of this study was to investigate these twin issues: i) the factors associated with late CLD diagnosis in a community cohort and ii) the impact of late diagnosis on subsequent survival - using data from the UK biobank ${ }^{13}$ study. 
METHODS:

\section{Participants}

UK biobank (UKB) is a community cohort study of 502,535 individuals aged 40-69 years in the UK. Study recruitment took place between May 2006 and July 2010 from 22 assessment centres covering all major areas of the UK. Participants completed a detailed health questionnaire and submitted blood samples for biomarker and host genotyping analysis. Follow-up data on major health outcome events are provided via record linkage to UK mortality, hospital and cancer registries ${ }^{13}$.

\section{Study cohort}

The study cohort included all UKB participants who presented for the first time with advanced CLD shortly after their UKB interview (i.e. within 5 years).

Presentation with advanced CLD was defined as either: a) a first time inpatient hospital admission for liver cirrhosis, or b) a first time diagnosis of HCC. Hospital admissions due to liver cirrhosis were identified using a validated definition previously described by Ratib et al. ${ }^{14}$ This cirrhosis definition comprises a combination of ICD 10 and OPCS4 codes enumerated in eTable 1. We included all admissions meeting this ICD cirrhosis definition, irrespective of whether they were elective/planned hospital admissions or emergency hospital admissions. Diagnoses of HCC were identified from national cancer registers in England, Wales and Scotland, using the ICD code: C22.0. Participants who had presented with advanced CLD prior to their UKB interview were excluded from this study. Inferring Late diagnosis

Progression to advanced CLD occurs slowly and is typically a product of decades of liver disease and inflammation ${ }^{15}$. Participants included in this study must all have had significant CLD at the time of their UKB interview therefore, by virtue of the fact that they all presented with advanced CLD within a short time (i.e. $<5$ years) of this date.

At their UKB interview, each participant was asked to report all "serious medical conditions" diagnosed by a doctor. This question was posed during a face-to-face interview with a trained nurse. 241 distinct medical conditions were reported by all UKB participants in total. We classified each diagnosis according to whether it represented a diagnosis of CLD or not. Full details of this classification are shown in Appendix A.

For the purpose of this analysis, individuals who reported a diagnosis of CLD were considered to have had a timely diagnosis of CLD. Vice versa, those who did not were considered to have received a late diagnosis of CLD.

Statistical analysis 
This study is based on two distinct, but complementary, analyses (see Supplementary Figure.1). The purpose of analysis \#1 was to identify individual-level factors associated with a late CLD diagnosis. Conversely, the purpose of analysis \#2 was to determine the association between a late CLD diagnosis and subsequent.

Analysis\#1: Individual-level factors associated with a late CLD diagnosis.

The following variables were considered in analysis \#1 as candidate predictors of late diagnosis: age at UKB interview, gender, Townsend deprivation score (as tertiles) ${ }^{16}$; birth outside of the UK; UK country of residence; main liver disease aetiology; platelet count; and stomach/abdominal pain for $>3$ months prior to UKB interview. Analogous to previous studies ${ }^{14}$, liver disease aetiology was ascertained through a hierarchical definition of: \#a) viral hepatitis, \#b) autoimmune liver disease in the absence of \#a; \#c) alcohol-related liver disease (ArLD) in the absence of \#a-\#b; \#d) non-alcoholic fatty liver disease (NAFLD) in the absence of \#a-\#c; and \#e) other/unknown in the absence of \#a-\#d. These aetiologies were discerned through a combination of hospital admissions and/or information reported during the survey interview (see eTable 2).

The crude association between each candidate predictors and late diagnosis was assessed using univariate logistic regression. Predictors variables with a univariate $\mathrm{p}$-value $<0.1$ were then included in a multivariable logistic regression model to identify independently associated factors.

Analysis\#2: Association between a late CLD diagnosis and survival.

We used survival analysis to assess the association between late diagnosis and post-advanced CLD mortality risk. We started follow-up time at the date of first advanced CLD event and ended it at the date of mortality (if at all), or the date the mortality registry was complete to (31 Jan 2018 for England and Wales participants; or 30 Nov 2016 for Scottish participants)(Supplementary Figure 1). We calculated the association between late CLD diagnosis and time to post-advanced CLD death using Cox regression models. We assessed this association according to tiered levels of covariate adjustment. These tiers were: a) No adjustment, b) Adjustment for sociodemographic factors (age; gender; birth outside UK; country of residence; and Townsend deprivation index), c) Adjustment for sociodemographic factors + aetiology, and d) Adjustment for sociodemographic +aetiology + disease stage-related variables (presence of ascites; renal dysfunction; infection; bleeding varices and HCC at index SLM; platelet count at UKB interview).

We investigated whether the magnitude of association between late diagnosis and mortality varied according to the following factors: aetiology, CLD severity, and follow-up time. This was done by adding appropriate interaction terms into the Cox regression model. To note, in this analysis, individuals with viral hepatitis, autoimmune hepatitis and other/unknown aetiologies were 
amalgamated into a single aetiology category due to the low number of deaths observed in these categories. CLD severity was defined in terms of whether one or more complication event was present at the index advanced CLD event. The complications we considered here were HCC, ascites, bleeding varices, infection and renal dysfunction. Two mutually exclusive follow-up time periods were considered: $<1$ year after incident advanced CLD, and $\geq 1$ year after incident advanced CLD.

In addition to those in the first analysis, this second analyses included presence of HCC at the index advanced CLD episode, presence of CLD-related complications at the incident advanced CLD event, defined using validated ICD10 codes (see eTable 3) ${ }^{1,17-20}$. 
RESULTS:

Study cohort

980 participants (0.2\%) developed advanced CLD within five years of UKB recruitment. Of these, 255 were excluded because an earlier advanced CLD event prior to UKB recruitment was identified.

Thus, our final sample comprised 725 participants, each of whom developed SLM for the first time within 5 years of their UKB interview. 93\% of these SLM events were related to hospital admissions for liver cirrhosis (see Supplementary Figure.2).

\section{Characteristics of study cohort}

The mean age at UKB recruitment was 59.8 years (sd:6.8), 489 (67\%) were male, and 61 (92\%) were born in the UK. The most common CLD aetiology was ArLD ( $\mathrm{n}=336,46 \%)$, followed by NAFLD ( $n=194,27 \%)$, viral hepatitis $(n=73,10 \%)$ and autoimmune liver disease $(n=72,10 \%) .51 \%$ of this cohort were in the most deprived tertile (versus $33 \%$ for the total UKB sample).

The mean time between UKB recruitment and incident SLM was 2.7 years and did not vary according to liver disease aetiology (see Supplementary Figure.3).

Late vs timely diagnosis

The timely diagnosis group comprised 122 (17\%) individuals reported at UKB recruitment that they had been diagnosed with a CLD-related condition. The most common condition indicated was "liver failure/liver cirrhosis” (see Supplementary Figure.4). The remaining 603 (83\%) did not indicate any knowledge of their CLD, and thus were assumed to have been diagnosed at a later point, which by definition, must have been very close to the time of advanced CLD_onset.

Analysis\#1: Individual-level factors associated with a late CLD diagnosis and post-advanced CLD mortality.

The strongest predictor of late diagnosis was liver disease aetiology (see Table 1 and Figure 1). The odds of late diagnosis were twelve times higher for an individual with ArLD versus viral hepatitis (aOR: 12.01;95\% CI: 6.32-22.83). Similarly, the odds of a late diagnosis were seven times higher for an individual with NAFLD versus viral hepatitis (aOR: 7.14;95\% CI:3.63-14.02).

Late diagnosis was also associated with symptoms and biomarkers. A platelet count of $<100$ was associated with 62\% lower odds of late diagnosis relative to a platelet count exceeding 150 (aOR: 0.38; 95\% CI: 0.18-0.76). The presence of abdominal pain was associated with reduced odds of late diagnosis (aOR: 0.49; 95\% CI:0.28-0.86). 
Participants were followed-up for 2927 person years post-advanced CLD. The mean follow-up duration per individual was 4.0 years (see Table 2). 342 deaths were observed, of which half had a liver-related cause (see Supplementary Figure.5). Cumulative mortality at 5 years post- advanced CLD was 27.4\% (95\% CI: 19.2-35.5) and 46.5\% (95\% CI: 42.4-50.6) for individuals with timely diagnosis and late diagnosis, respectively (see Figure 2).

Analysis\#2: Association between a late CLD diagnosis and survival.

Late diagnosis was significantly associated with a higher post- advanced CLD mortality risk in univariate analysis (HR:1.47: 95\% CI: 1.08-2.00; $\mathrm{p}=0.013$ ), but the association attenuated after adjustment for covariates (see Table 3).

In subgroup analyses, the association between late CLD diagnosis and post- advanced CLD mortality varied according to aetiology. It was greater for patients with NAFLD (aHR: 2.18; 95\% CI: 0.86-5.51; $\mathrm{P}=0.100$ ) versus patients with ArLD (aHR: 0.82; 95\% CI: 0.48-1.40). The p-value for interaction however, at 0.11-0.19, was not statistically significant (see Figure.3). Conversely, there was no sign of effect modification with respect to either follow-up time or CLD severity after adjustment for covariates (see Supplementary Figure.6-7). 


\section{DISCUSSION}

Main findings of this study

Previous studies have demonstrated that many patients do not receive a diagnosis of CLD until after the onset of advanced CLD ${ }^{3,10,11,21}$. Our current understanding of the determinants of this phenomenon remains limited, especially in the context of a community cohort. We have exploited a unique opportunity to investigate this issue by studying the data of 725 UKB participants that developed advanced CLD for the first time shortly after their recruitment interview.

\section{What this study adds}

Foremost, our results confirm that late CLD diagnosis is commonplace, applicable to more than three-quarters of patients with advanced CLD. However, we have also extended previous work by showing that the odds of a late diagnosis vary according to clinical factors. In particular, we found that late diagnosis was strongly influenced by liver disease aetiology; specifically, the odds of a late diagnosis were twelve times (aOR: 12.01; $\mathrm{p}<0.001$ ) greater for an individual with ArLD versus an individual with viral hepatitis. Similarly, the odds of late diagnosis were seven times greater (aOR: 7.14; $\mathrm{P}<0.001$ ) for an individual with NAFLD versus viral hepatitis.

This “aetiology disparity” could reflect a number of factors, including: i) reluctance of general practitioners (GPs) to initiate liver disease investigation for patients with ArLD/NAFLD risk factors because of a perception that a diagnosis would be futile (i.e. would not change care plan given a perception that specialist therapeutic options are limited) ${ }^{22}$; or ii) more complex risk stratification algorithms for NAFLD and ArLD that GPs are not familiar with and/or have difficulty interpreting ${ }^{22-}$ ${ }^{24}$. We also found that the odds of a late diagnosis were reduced for individuals with a low platelet count and with abdominal pain. This is presumably because these clinical features trigger further investigations, that subsequently reveal evidence of CLD. However, most patients had neither of these clinical "red flags", which emphasises the challenge of identifying high risk of advanced CLD patients ex ante. Overall, we confirm the limited and suboptimal performance of existing CLD diagnostic pathways in community settings. Future research should endeavour to improve these pathways - especially for patients with ArLD and NAFLD.

A second objective of this study was to explore whether late diagnosis is associated with impaired post- advanced CLD survival. This might be the case if, for example, non-late diagnosis patients were better primed/prepared than late diagnosis patients to adopt and maintain health behaviour changes following onset of advanced CLD. We found that late diagnosis patients did have a considerably higher five-year cumulative mortality than timely diagnosed patients (see Figure 2) - however, after adjustment for relevant covariates, the survival differences were attenuated and a significant effect did 
not persist (see Table 3). This observation could reflect either limited statistical power or a true null effect. Interestingly however, our subgroup analysis suggests that the impact of a late diagnosis may vary according to aetiology (see Figure $\underline{3} 4$ ). I.e. specifically, a late diagnosis appeared to be much more detrimental to post- advanced CLD survival for patients with NAFLD (aHR: 2.18; 95\% CI: 0.86-5.51; $\mathrm{P}=0.10$ ) versus patients with ArLD (aHR: 0.82; 95\% CI: 0.48-1.40). Future research should explore this finding in greater detail and - if possible -with a greater sample size. This is important firstly, because the burden of NAFLD is expected to increase considerably in the next decade ${ }^{25,26}$. Secondly, because the combination of a high underlying mortality rate plus a high prevalence of late diagnosis, means that even a very modest association would translate into an appreciable number of life years lost. Thirdly because a better understanding of the harms and disbenefits of a late diagnosis (in terms of hard clinical endpoints) will conceivably encourage further investment in CLD detection initiatives.

\section{Limitations of this study}

Late diagnosis is an important issue in Hepatology, but it is understudied, meaning that basic questions remain unanswered. The key strength of this study is that we have been able to address some of these questions that have been inadequately explored thus far. Our study does have several limitations that require note. The first limitation is that we inferred late diagnosis from self-reported data; the accuracy of these self-reported data could have been affected by recall bias, "interview fatigue” or under-reporting as a result of stigma. However, although stigma varies according to liver disease aetiology, we think this is very unlikely to account for the observed "aetiology disparity" because stigma is comparably high for viral hepatitis and $\operatorname{ArLD}^{27}$, and yet we observed diametrically opposite levels of CLD diagnosis between these two groups. Furthermore, we also checked selfreported CLD diagnosis for 598 UKB participants with advanced CLD prior to their UKB interview i.e. a "negative control” group that one would assume would all have received a diagnosis of CLD. Reassuringly, self-reported diagnosis was vastly higher in this negative control group across all aetiologies (see Supplementary Figure.8), but nevertheless it was still short of 100\% (69\% overall). The shortfall may reflect misclassification of CLD diagnosis status, but potentially suboptimal communication of liver disease to patients as well ${ }^{28-30}$. A second limitation is that the UKB sample are not representative of the general UK population; on average, UKB participants are more likely to be female, older in age, and to live in less socioeconomically deprived areas than non-participants ${ }^{31}$. We are unclear therefore about how generalizable these findings will be to the rest of the UK, and to settings outside the UK. Thirdly, we did not have access to data on specific treatments received following first cirrhosis hospitalisation, which may have shed more light on the association between late CLD diagnosis and subsequent prognosis. Fourth, UKB participants were recruited in 2006-2010 (median recruitment date was 2009). Thus the situation we report here regarding late diagnosis may have subsequently changed, particularly since the publication of new CLD detection guidelines by 
NICE $^{32,33}$ and the British Society of Gastroenterology ${ }^{34}$ from 2016. Finally, when assigning individuals to viral hepatitis and autoimmune aetiologies, we did not have direct data on appropriate blood test results (e.g. HCV antibodies, and autoantibodies), but had to rely instead on ICD codes recorded in hospital episodes (see eTable 1).

Early diagnosis and risk stratification are regarded as being key to improving the prognosis of patients with $\mathrm{CLD}^{3,35,36}$. Health care professionals should ensure that risk factors are identified and followed up at all contacts. Training outside of general practice may be needed as frequently people with hazardous alcohol risk factors present to emergency health and social care settings ${ }^{37}$, from which a process for referral for liver assessment needs to be supported. Furthermore, patients with NAFLD related risk factors (e.g. type 2 diabetes) are known to have frequent community or secondary care attendances so there are likely to be ample opportunities for risk assessment. Not only is there opportunity to identify patients at an earlier stage, but there is an economic necessity as well, given the huge cost managing patients with advanced chronic liver disease ${ }^{38,39}$.

In conclusion, late CLD diagnosis is commonplace in a community cohort setting, but varies markedly according to aetiology. Our results confirm the need for more action at the general population/community level to improve early detection of $\mathrm{CLD}^{3,40}$. This is especially true for ArLD and NAFLD.

\section{ACKNOWLEDGEMENTS}

Conflict of interests: Nothing to disclose

Financial support: We acknowledge support from Glasgow Caledonian University core research funding; Health Protection Scotland; NIHR Nottingham Biomedical Research Centre; and UK Medical Research Council (JRM receives salary support from an MRC Clinician Scientist Award $\mathrm{MR} / \mathrm{P008348/1).}$

Authors contributions: a) Study concept: all authors; b) study design: all authors: c) acquisition of data: all authors; d) Statistical analysis: HI; e) drafting manuscript: HI; f) critical revision of manuscript: all authors. 


\section{REFERENCES}

1. Wang, H. et al. Global, regional, and national life expectancy, all-cause mortality, and causespecific mortality for 249 causes of death, 1980-2015: a systematic analysis for the Global Burden of Disease Study 2015. The lancet 388, 1459-1544 (2016).

2. Williams, R. et al. Disease burden and costs from excess alcohol consumption, obesity, and viral hepatitis: fourth report of the Lancet Standing Commission on Liver Disease in the UK. The Lancet 391, 1097-1107 (2018).

3. Williams, R. et al. Addressing liver disease in the UK: a blueprint for attaining excellence in health care and reducing premature mortality from lifestyle issues of excess consumption of alcohol, obesity, and viral hepatitis. The Lancet 384, 1953-1997 (2014).

4. Ratib, S., Fleming, K. M., Crooks, C. J., Aithal, G. P. \& West, J. 1 and 5 year survival estimates for people with cirrhosis of the liver in England, 1998-2009: a large population study. J. Hepatol. 60, 282-289 (2014).

5. Ruhl, C., Sayer, B., Byrd-Holt, D. \& Brown, D. Costs of digestive diseases. Burd. Dig. Dis. U. S. US Dep. Health Hum. Serv. Public Health Serv. Natl. Inst. Health Natl. Inst. Diabetes Dig. Kidney Dis. Wash. DC US Gov. Print. Off. 142, (2008).

6. Ratib, S., Fleming, K. M., Crooks, C. J., Walker, A. J. \& West, J. Causes of Death in People with Liver Cirrhosis in England Compared with the General Population: A Population-Based Cohort Study. Am. J. Gastroenterol. 110, (2015).

7. UK Department of Health. Advancing our health: prevention in the 2020s. (2019).

8. NHS. The NHS Long Term Plan. (2019).

9. Hydes, T., Gilmore, W., Sheron, N. \& Gilmore, I. Treating alcohol-related liver disease from a public health perspective. J. Hepatol. 70, 223-236 (2019).

10. Samji, H. et al. Late hepatitis B and C diagnosis in relation to disease decompensation and hepatocellular carcinoma development. J. Hepatol. 67, 909-917 (2017).

11. Chirikov, V. V., Shaya, F. T. \& Howell, C. D. Contextual analysis of determinants of late diagnosis of hepatitis C virus infection in medicare patients. Hepatology 62, 68-78 (2015). 
12. Armstrong, M. J. et al. Presence and severity of non-alcoholic fatty liver disease in a large prospective primary care cohort. J. Hepatol. 56, 234-240 (2012).

13. Sudlow, C. et al. UK biobank: an open access resource for identifying the causes of a wide range of complex diseases of middle and old age. PLoS Med. 12, (2015).

14. Ratib, S., West, J., Crooks, C. J. \& Fleming, K. M. Diagnosis of Liver Cirrhosis in England, a Cohort Study, 1998-2009: A Comparison With Cancer. Am J Gastroenterol 109, 190-198 (2014).

15. Schuppan, D. \& Afdhal, N. H. Liver cirrhosis. The Lancet 371, 838-851 (2008).

16. Townsend, P., Phillimore, P. \& Beattie, A. Health and deprivation: inequality and the North. (Routledge, 1988).

17. D’Amico, G., Garcia-Tsao, G. \& Pagliaro, L. Natural history and prognostic indicators of survival in cirrhosis: a systematic review of 118 studies. J. Hepatol. 44, 217-231 (2006).

18. Fede, G. et al. Renal failure and cirrhosis: a systematic review of mortality and prognosis. $J$. Hepatol. 56, 810-818 (2012).

19. Arvaniti, V. et al. Infections in patients with cirrhosis increase mortality four-fold and should be used in determining prognosis. Gastroenterology 139, 1246-1256 (2010).

20. Asrani, S. K. et al. Trends in chronic liver disease-related hospitalizations: a population-based study. Am. J. Gastroenterol. 114, 98-106 (2019).

21. Scaglione, S. et al. The epidemiology of cirrhosis in the United States. J. Clin. Gastroenterol. 49, 690-696 (2015).

22. Standing, H. C. et al. GPs' experiences and perceptions of early detection of liver disease: a qualitative study in primary care. Br J Gen Pr. 68, e743-e749 (2018).

23. Patel, P. J. et al. Underappreciation of non-al coholic fatty liver disease by primary care clinicians: limited awareness of surrogate markers of fibrosis. Intern. Med. J. 48, 144-151 (2018).

24. Marjot, T. et al. Prevalence and severity of non-al coholic fatty liver disease are underestimated in clinical practice: impact of a dedicated screening approach at a large university teaching hospital. Diabet. Med. 35, 89-98 (2018). 
25. Estes, C., Razavi, H., Loomba, R., Younossi, Z. \& Sanyal, A. J. Modeling the epidemic of nonalcoholic fatty liver disease demonstrates an exponential increase in burden of disease. Hepatology 67, 123-133 (2018).

26. Estes, C. et al. Modeling nafld disease burden in china, france, germany, italy, japan, spain, united kingdom, and united states for the period 2016-2030. J. Hepatol. 69, 896-904 (2018).

27. Vaughn-Sandler, V., Sherman, C., Aronsohn, A. \& Volk, M. L. Consequences of perceived stigma among patients with cirrhosis. Dig. Dis. Sci. 59, 681-686 (2014).

28. Goldsworthy, M. A. et al. Patient understanding of liver cirrhosis and improvement using multimedia education. Frontline Gastroenterol. 8, 214-219 (2017).

29. NCEIPOA, D. Measuring the Units: A review of patients who died with alcohol related liver disease. (2013).

30. Low, J. T. et al. Supportive and palliative care in people with cirrhosis: international systematic review of the perspective of patients, family members and health professionals. J. Hepatol. 69, 1260-1273 (2018).

31. Fry, A. et al. Comparison of sociodemographic and health-related characteristics of UK Biobank participants with those of the general population. Am. J. Epidemiol. 186, 1026-1034 (2017).

32. NICE guidelines [NG49] Non-alcoholic fatty liver disease (NAFLD): assessment and management. (National Institute for Health and Care Excellence (NICE), 2016).

33. NICE guidelines [NG50] Cirrhosis in over 16s: assessment and management. (National Institute for Health and Care Excellence (NICE), 2016).

34. Newsome, P. N. et al. Guidelines on the management of abnormal liver blood tests. Gut 67, 6-19 (2018).

35. Askgaard, G., Neermark, S., Leon, D. A., Kjær, M. S. \& Tolstrup, J. S. Hospital contacts with alcohol problems prior to liver cirrhosis or pancreatitis diagnosis. World J. Hepatol. 9, 13321339 (2017).

36. Askgaard, G., Kjær, M. S. \& Tolstrup, J. S. Opportunities to Prevent Alcoholic Liver Cirrhosis in High-Risk Populations: A Systematic Review With Meta-Analysis. Am. J. Gastroenterol. 114, (2019). 
37. Balp, M.-M. et al. The burden of non-alcoholic steatohepatitis (NASH) among patients from Europe: A real-world patient-reported outcomes study. JHEP Rep. 1, 154-161 (2019).

38. Bouttell, J. et al. The SCottish Alcoholic Liver disease Evaluation: A Population-Level Matched Cohort Study of Hospital-Based Costs, 1991-2011. PLOS ONE 11, e0162980 (2016).

39. Younossi, Z. M. \& Henry, L. Economic and quality-of-life implications of non-alcoholic fatty liver disease. Pharmacoeconomics 33, 1245-1253 (2015).

40. Ginès, P. et al. Screening for liver fibrosis in the general population: a call for action. Lancet Gastroenterol. Hepatol. 1, 256-260 (2016). 


\section{TABLES AND FIGURES}

Figure 1. Odds of late diagnosis, by aetiology (relative to viral hepatitis)

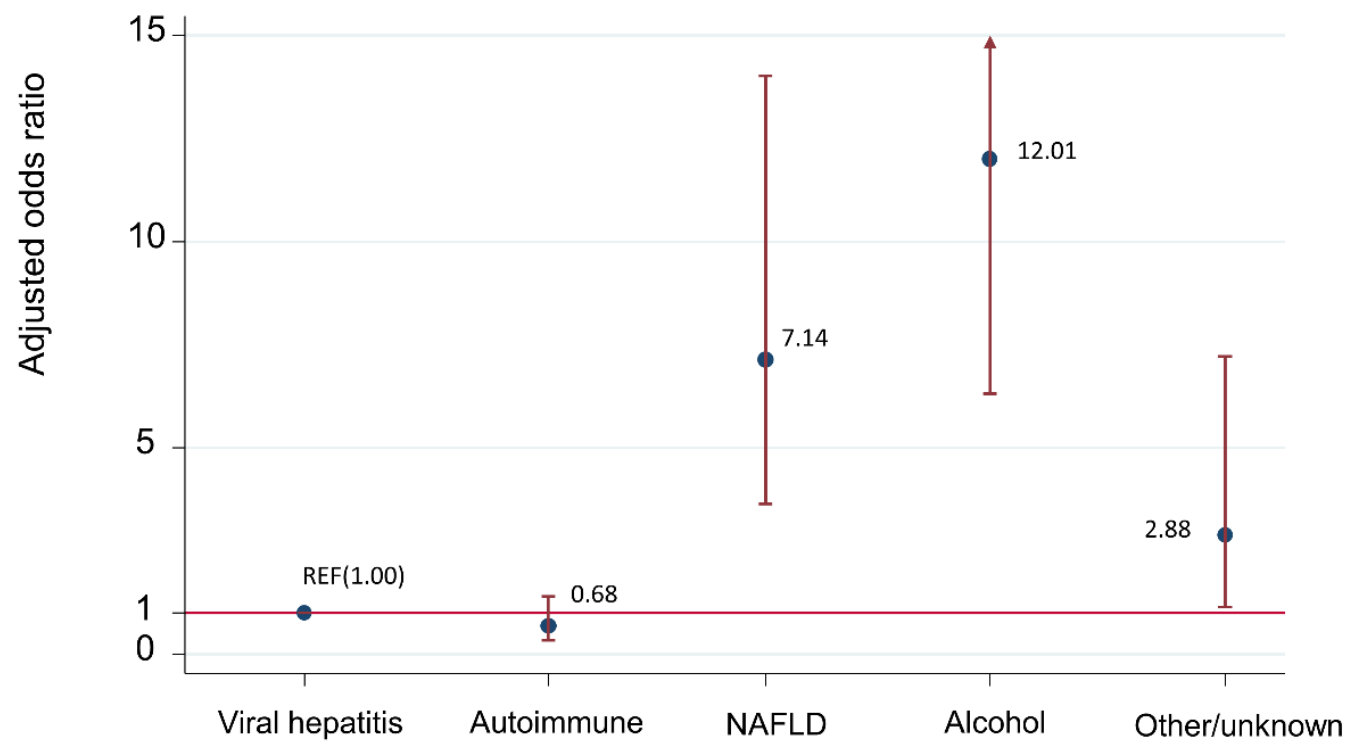

Aetiology 
Figure 2. Crude survival after the index advanced CLD event, according to late diagnosis status.

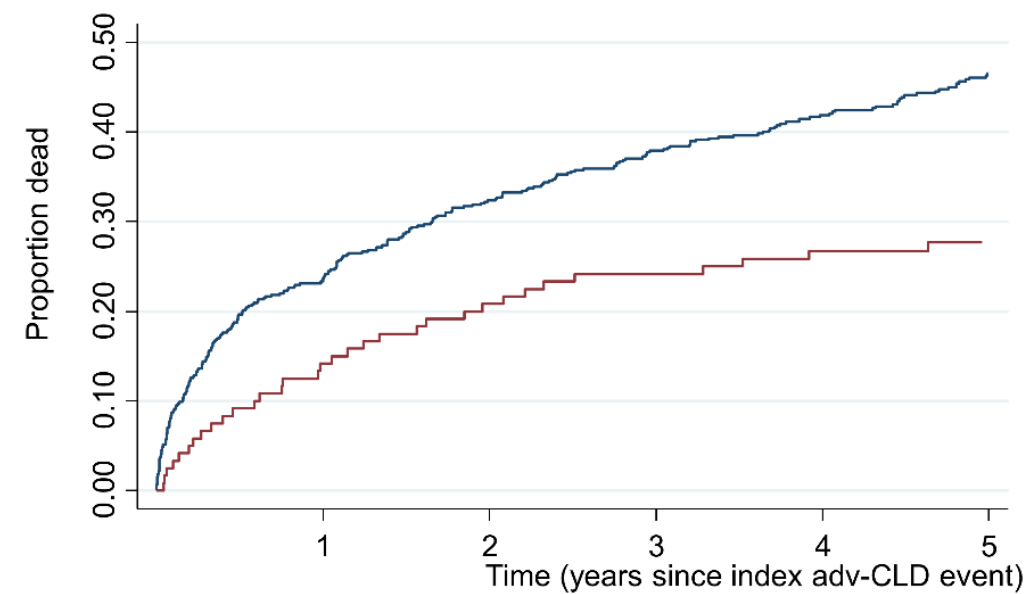

Number at risk

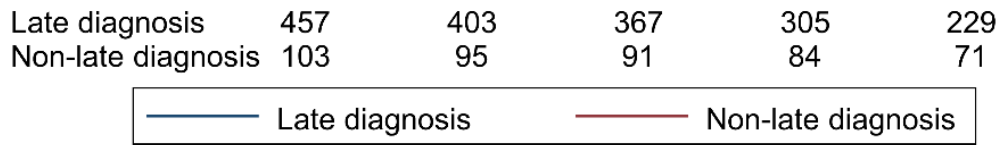

Figure 3. Association between late diagnosis and survival following index advanced CLD event, according to aetiology

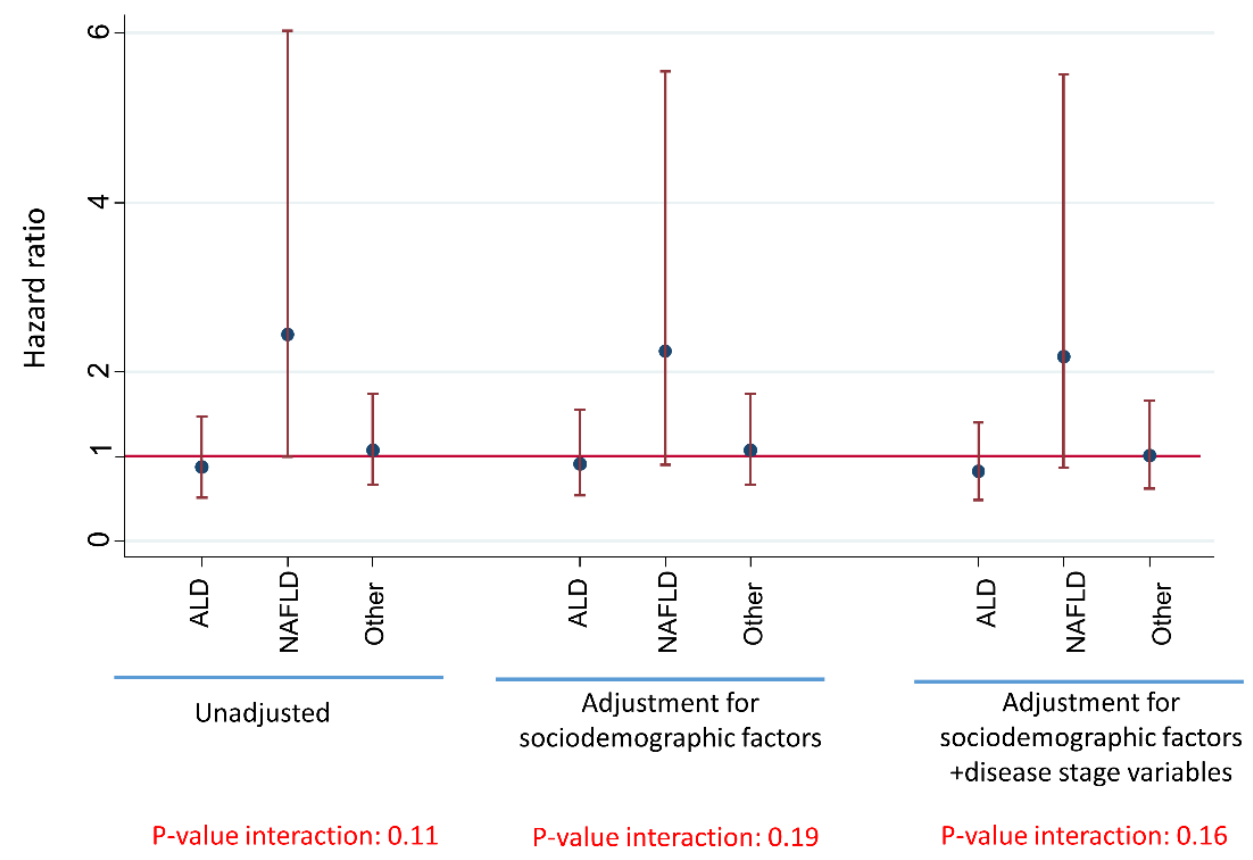



Table 1: Cohort description and factors associated with late chronic liver disease diagnosis.

\begin{tabular}{|c|c|c|c|c|c|}
\hline \multirow[t]{2}{*}{ Participant characteristics $†$} & & \multirow{2}{*}{$\begin{array}{l}\text { Number of participants } \\
\text { (col_\%) }\end{array}$} & \multirow{2}{*}{$\begin{array}{l}\text { Late diagnosis } \\
\text { (row_\%) }\end{array}$} & \multicolumn{2}{|c|}{ Association: Odds ratio $(95 \% \mathrm{CI}) \ddagger$} \\
\hline & & & & Univariate & Multivariate \\
\hline \multirow[t]{4}{*}{ Age group, years } & $40-54$ & $175(24.1)$ & $141(80.6)$ & REF (1.00) & \multirow[t]{4}{*}{ N/A } \\
\hline & $55-59$ & $139(19.2)$ & $110(79.1)$ & $0.91(0.53-1.59)$ & \\
\hline & $60-64$ & 212 (29.2) & $179(84.4)$ & $1.31(0.77-2.22)$ & \\
\hline & $65+$ & 199 (27.5) & 173 (86.9) & $1.60(0.92-2.80)$ & \\
\hline \multirow[t]{2}{*}{ Gender } & Male & 489 (67.5) & 417 (85.3) & REF (1.00) & \multirow{2}{*}{$\begin{array}{l}\operatorname{REF}(1.00) \\
1.07(0.64-1.79)\end{array}$} \\
\hline & Female & $236(32.6)$ & $186(78.8)$ & $0.64(0.43-0.96)^{*}$ & \\
\hline \multirow{3}{*}{$\begin{array}{l}\text { Townsend deprivation } \\
\text { index }\end{array}$} & 1 (least deprived) & $168(23.2)$ & $140(83.3)$ & REF (1.00) & \multirow{3}{*}{$\mathrm{N} / \mathrm{A}$} \\
\hline & 2 & $186(25.7)$ & 156 (83.9) & 1.04 (0.59-1.83) & \\
\hline & 3 (most deprived) & $371(51.2)$ & 307 (82.8) & $0.96(0.59-1.56)$ & \\
\hline \multirow[t]{2}{*}{ Born outside UK } & No & $664(91.6)$ & $556(83.7)$ & REF (1.00) & \multirow{2}{*}{$\mathrm{N} / \mathrm{A}$} \\
\hline & Yes & $61(8.4)$ & $47(77.1)$ & $0.65(0.35-1.23)$ & \\
\hline \multirow[t]{2}{*}{ Resident country } & England/Wales & $669(92.3)$ & $557(83.3)$ & REF (1.00) & \multirow{2}{*}{$\mathrm{N} / \mathrm{A}$} \\
\hline & Scotland & $56(7.7)$ & $46(82.1)$ & $0.92(0.45-1.89)$ & \\
\hline \multirow[t]{5}{*}{ Liver disease etiology } & Viral hepatitis & $73(10.1)$ & $38(52.1)$ & REF (1.00) & REF (1.00) \\
\hline & Autoimmune & $72(9.9)$ & 35 (48.6) & $0.87(0.45-1.67)$ & $0.68(0.33-1.40)$ \\
\hline & Alcohol & $336(46.3)$ & $314(93.5)$ & $13.15(7.00-24.7)^{* *}$ & $12.01(6.32-22.83) * *$ \\
\hline & NAFLD & $194(26.8)$ & $175(90.2)$ & $8.48(4.39-16.41)^{* *}$ & $7.14(3.63-14.02)^{* *}$ \\
\hline & other/unknown & $50(6.9)$ & $41(82.0)$ & $4.20(1.78-9.87)^{*}$ & $2.88(1.15-7.21)^{*}$ \\
\hline \multirow[t]{4}{*}{ Platelet count, $\times 10^{9} / \mathrm{L}$} & $\geq 150$ (normal) & $487(67.2)$ & $415(85.2)$ & REF (1.00) & REF (1.00) \\
\hline & $100-149$ & $138(19.0)$ & $114(82.6)$ & $0.82(0.50-1.37)$ & $0.78(0.44-1.39)$ \\
\hline & $<100$ (low) & $59(8.1)$ & $39(66.1)$ & $0.34(0.19-0.61)^{* *}$ & $0.38(0.18-0.76)^{*}$ \\
\hline & missing & $41(5.7)$ & $35(85.4)$ & $1.01(0.41-2.49)$ & $1.48(0.54-4.03)$ \\
\hline \multirow{2}{*}{$\begin{array}{l}\text { Adominal/stomach pain in } \\
\text { last month }\end{array}$} & No & 604 (83.3) & $511(84.6)$ & REF (1.00) & REF (1.00) \\
\hline & Yes & $121(16.7)$ & $92(76.0)$ & $0.58(0.36-0.93)^{*}$ & $0.56(0.32-0.97)^{*}$ \\
\hline TOTAL & & $725(100.0)$ & $603(83.2)$ & - & - \\
\hline
\end{tabular}

$\dagger$ measured at UKB interview

‡ derived via logistic regression. Variable selection criteria for multivariate analysis was p-value $<0.10$ in univariate analysis. Variables that did not meet this criteria are marked "NA" in multivariate column

$*$ denotes p-val $\leq 0.05$; ** denotes p-value $\leq 0.001$ 
Table 2. Description of follow-up data.

\begin{tabular}{lccc} 
& \multicolumn{2}{c}{ Late diagnosis of CLD } & No \\
\hline Total persons & Yes & 122 & 725 \\
total person years follow-up & 603 & 594 & 2927 \\
Average years of follow up, per person (mean) & 2333 & 4.9 & 4.0 \\
Average years of follow up, per person (median & 3.9 & 5.5 & 4.3 \\
Number of all-cause deaths & 4.1 & 48 & 342 \\
crude all cause mortality rate, per 100 PYs (95\% CI) & $12.6(11.2-14.1)$ & $8.1(6.1-10.7)$ & $11.7(10.5-13.0)$ \\
\hline
\end{tabular}


Table 3: Association between late diagnosis and survival following index CLD event, according to different levels of covariate adjustment

\begin{tabular}{llc} 
Model \# (variables adjusted for) & $\begin{array}{l}\text { HR for late diagnosis versus } \\
\text { non-late diagnosis (95\% CI) }\end{array}$ & P-value \\
\hline Model \#1 (univariate - no adjustment) & $1.47(1.08-2.00)$ & 0.013 \\
Model \#2 (socio demographic factors ${ }^{1}$ ) & $1.35(0.99-1.84)$ & 0.056 \\
Model \#3 (as per model\#2 + liver disease aetiology) & $1.11(0.79-1.55)$ & 0.550 \\
Model \#4 (as per model \#3 + disease stage ${ }^{2}$ ) & $1.04(0.74-1.48)$ & 0.811 \\
\hline
\end{tabular}

1 . Sociodemographic factors comprises: age, gender and birth outside UK; UK resident country; and Townsend deprivation index.

2. disease stage comprises: platelet count at UKB interview, presence of bleeding varices at index advanced CLD event, chronic kidney disease at index advanced CLD event, ascites at index advanced CLD event, infection at index advanced CLD event. 
